\title{
Fisher Discriminant Analysis of EEG Data based on Drinking Events
}

\author{
Yuan Shi* \\ School of Soft Engineering, Dalian Institute of Science and Technology, Dalian, Liaoning, 116028, P.R. China
}

\begin{abstract}
Objective: We have performed Fisher's discriminant analysis to the EEG data of experimental subjects who were drunk on alcohol. The subjects of these experiments were recorded anonymously. The data has been used for feature extraction and classification decisions in order to determine the part of head electrode's categories after alcohol intake and to explore the changes of EEG features. The calculation methods have been divided into four species In accordance with the strength of $\alpha$ wave. The head electrodes have been used as a part of of 21 electrodes EEG data used for 6 subjects. We have performed Fisher's discriminant analysis of the EEG data on the six subjects. After every 20 minutes $7.2 \mathrm{ml}$ of alcohol was given to the subjects for drinking. The EEG data processing and statistical analysis adopted was independently designed regarding the EEG analysis toolbox and the program for correlation analysis. Results of the Fisher discriminant would be better applied to the feature extraction and classification decisions of the EEG data. Conclusions: EEG activity shows a significant response after alcohol intakes, electrode categories is noted to constantly change. After drinking $200 \mathrm{ml}$ categories changes obviously, and drinking $600 \mathrm{ml}$ category changes become calm. The changes found are not so obvious in men but are significantly obvious in women.
\end{abstract}

Keywords: Drinking volume, electroencephalogram, fisher discriminant analysis.

\section{INTRODUCTION}

The purpose of routine brain wave inspection is to evaluate whether the brain wave is normal or not and provide help in diagnosing any brain disorders which are also known as brain wave interpretations [1]. The traditional brain wave interpretation is realized through reading the multi-channel EEG on the recording paper by experts, who try to understand and evaluate electroencephalogram (EEG) through visual inspection. The essence of this method based on expertise is that experts utilize experience to wipe out any disturbance and anomalies of the signals, conduct feature extraction of the EEG according to the frequency, range, phase position and other information, and carry out the category description of the extracted features with their experience to analyze and evaluate the EEG [2]. Up till now, this method is widely applied in clinic. The visual inspection, to some extent, can catch the pathological waveform or even confirm the position of the brain's focus. However, due to the strong non-stationary and nonlinear characteristics of the EEG, with the addition of the great dependence of visual inspection on knowledge-level and experience of EEG analysis personnel, the new method must be explored to realize the breakthrough of EEG research [3]. Bayes Discriminant analysis has been introduced in the EEG research. This will actively promote the extraction and classification of EEG data to assist the inspection and quantitative analysis of EEG and provide an effective analysis and means for the EEG examination.

\section{OBJECTS AND METHODS}

\subsection{Object of the Study}

We took 28 men and 35 women as the research subjects. The age of the subjects ranged from 20 to 60 years, with the average age being 36.7. All the subjects were in good health without serious nerve system diseases or any history of psychotropic drugs, and they were selected from normal population.

At the start of the experiment 6 subjects were taken, they were asked to be in peace with their eyes closed. At this time they had not been given any alcohol. Then they were given $200 \mathrm{ml}(3.6 \%$ alcohol $)$ in the form of beer and their EEG data were recorded after every 20 minutes, overall 3 records were taken. At the end of the experiment each person had consumed $600 \mathrm{ml}$ beer with $21.6 \mathrm{ml}$ alcohol. The number of sampling points for each EEG data acquired was 512, and each block took 5.12 seconds. Effective experimental events per subjects were 6: starting with no drinking, then drinking $200 \mathrm{ml}$ beer every 20 minutes for 3 times -3 experimental events of taking $7.2 \mathrm{ml}$ alcohol every 20 minutes.

\subsection{Build the Selection of Mathematical Modelling EEG Data}

The sampling frequency of experiment recording of EEG is $100 \mathrm{~Hz}$ which is recording 21 electrode data according to the lead location in international 10-20 system: $\mathrm{C}_{3}, \mathrm{C}_{Z}, \mathrm{C}_{4}$, $\mathrm{F}_{\mathrm{P} 1}, \mathrm{~F}_{\mathrm{PZ}}, \mathrm{F}_{\mathrm{P} 2}, \mathrm{~F}_{7}, \mathrm{~F}_{8}, \mathrm{~F}_{\mathrm{Z}}, \mathrm{F}_{3}, \mathrm{~F}_{4}, \mathrm{O}_{1}, \mathrm{O}_{\mathrm{Z}}, \mathrm{O}_{2}, \mathrm{P}_{3}, \mathrm{P}_{\mathrm{Z}}, \mathrm{P}_{4}, \mathrm{~T}_{5}, \mathrm{~T}_{6}$, $\mathrm{T}_{3}, \mathrm{~T}_{4}$ [4]. A block (indicating a short time period) of EEG data is acquired at every turn, and the number of sampling points for each block is 512 with the recording time of $5.12 \mathrm{~s}$ [5]. The electroencephalogram of normal people is usually in 
$\alpha$ rhythm, the strengths of wave appear in the occiput, and then weaken gradually from back to front. The 21 conducting electrodes can be classified into 4 categories in accordance with the intensity differences of the $\alpha$ rhythm in various parts of the head which are, former head electrode, side head electrode, central electrode, occiput electrode. The specific classification situation is as $\alpha$ follows [6]:

(1) The first category: central electrode $\left(\mathrm{C}_{3}, \mathrm{C}_{\mathrm{Z}}, \mathrm{C}_{4}\right)$

(2) The second category: former head electrode $\left(\mathrm{F}_{\mathrm{P} 1}, \mathrm{~F}_{\mathrm{PZ}}\right.$, $\mathrm{F}_{\mathrm{P} 2}, \mathrm{~F}_{7}, \mathrm{~F}_{8}, \mathrm{~F}_{\mathrm{Z}}, \mathrm{F}_{3}, \mathrm{~F}_{4}$ )

(3) The third category: occiput electrode $\left(\mathrm{O}_{1}, \mathrm{O}_{\mathrm{Z}}, \mathrm{O}_{2}, \mathrm{P}_{3}\right.$, $\left.\mathrm{P}_{\mathrm{Z}}, \mathrm{P}_{4}, \mathrm{~T}_{5}, \mathrm{~T}_{6}\right)$

(4) The forth category: side head electrode $\left(T_{3}, T_{4}\right)$

\subsection{The Computer Processing of EEG Data}

In order to facilitate the analysis of the raw data of the EEG, MATLAB programming is used to design a dedicated EEG Toolbox. In EEG Toolbox when raw data is accessed and saved in matrix, the horizontal lines represent the time points of lab records (namely sampling points) and the vertical columns stand for electrodes. Before analysis, all data of every subject should be accessed in EEG Toolbox and there should be a visual EEG, with one page including a block of EEG data.

In accordance with the electrode classification method described above, overall four Fisher's discriminant analyses divide sample data into four categories. First, according to the classification we will put the 21 electrode EEG data which has mathematical models into 4 matrixes $\bar{X}_{i}(i=1,2 \ldots 4)$. Then we put EEG data of the current blocks into the matrix X, whose measurement is $512 \times 21$. Now we will use Fisher discrimination analysis to predict the electrode classification results, and the results should be displayed in the form of the vector [7].

Fisher discrimination analysis will build a linear classifier surface in the feature space to project the space sample points along this direction, and identify categories

The predicted Classification Results of Fisher's discriminant analysis of the 6 events vary from each other, we only show the Classification Results of one person, with 2 blocks to demonstrate the whole. As rest of them are similar. Figs. (1 and 2) Illustrate the Fisher discriminant analysis of 2 blocks for the 6 subject in peace and with their eyes closed (event 1).

According to the values of these projections in its direction. Fisher discrimination analysis procedures in this research program are based on the multi-channel EEG data. First we must establish mathematical models, namely the discrimination functions, then predict the EEG data categories according to the discrimination rules. Fisher's discrimination analysis can be explained by the following mathematical formula:

$y=u^{\prime} X$

In the formula (1), $u$ is the feature vector corresponding to the maximum characteristic root of each $E^{-1} A$. Multi-

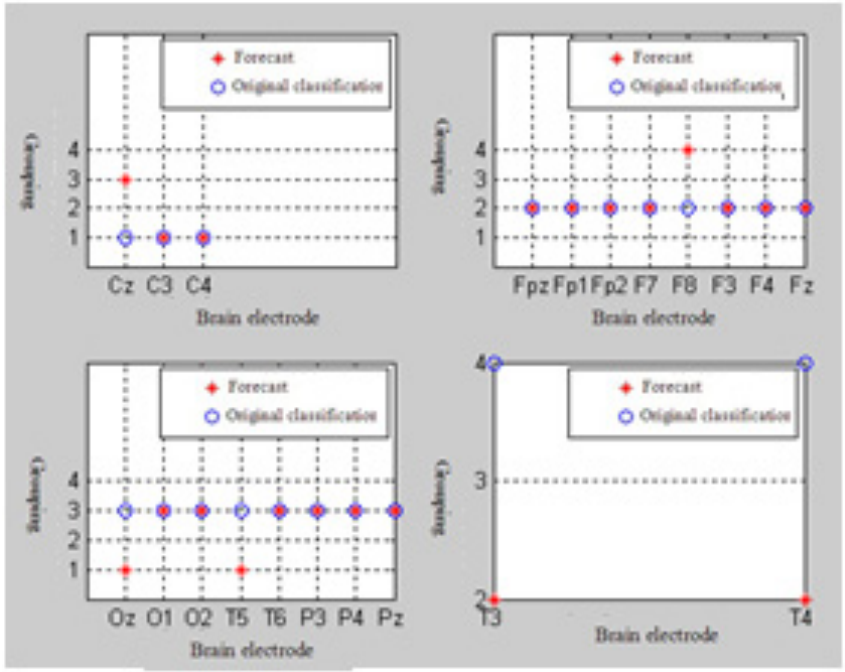

Fig. (1). Fisher results of the sixth subject in Event 1.

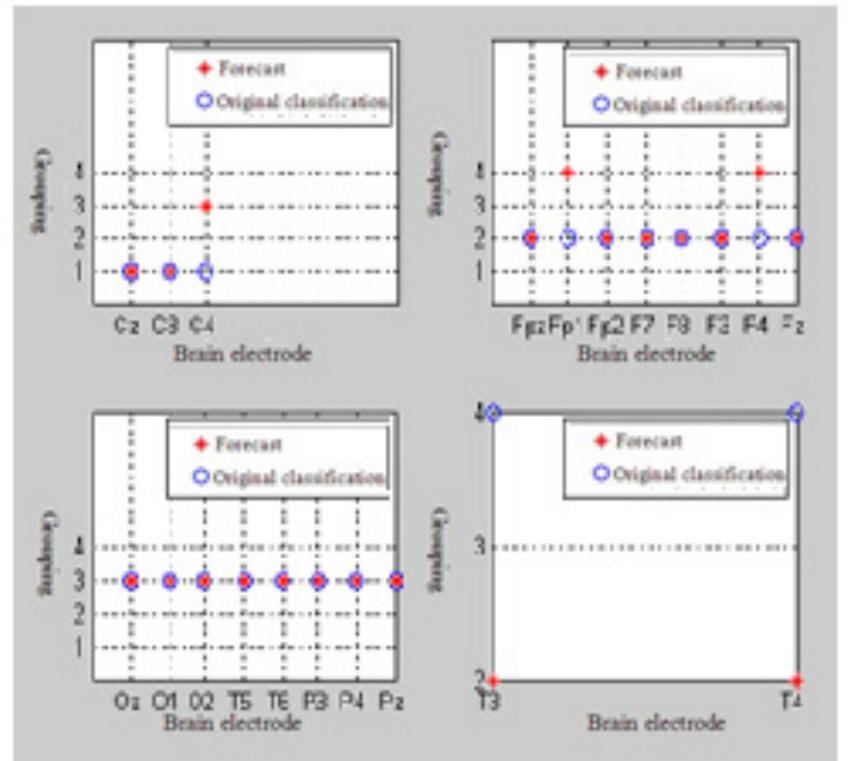

Fig. (2). Fisher results of the sixth people in Event 1.

dimensional discrimination needs to use many feature vectors in order to form multiple discriminant functions. We put unclassified EEG data $\mathrm{X}$ into four Fisher discriminant functions, and get the minimum distance and then match it with the corresponding whole. We can use Fisher discriminant functions to predict classifications of the EEG data of each block, and shows the predicted classification of the results, visually combined with the actual classification in Fisher discriminant analysis prediction map [8].

\section{EEG DATA ANALYSIS RESULTS FROM FISHER DISCRIMINANT}

The classification results of the 6 subjects from block 2 in the $1^{\text {st }}$ event were used to demonstrate the whole results, as the results from the rest blocks were similar. Figs. ( 1 and 2) illustrate the Fisher discriminant analysis of the 6 subjects in event 1: In the first category the central area was classified as the back of the head; In the second category, part of electrodes of the front of head was classified as the side of the 
head; in the third category, part of the electrodes of back of the head were classified as the central area; in the fourth category, all the electrodes of the side of the head were classified as the front of the head. The misjudgments normally happened when $\alpha$ wave is close to categories caused by the nonlinear characteristics of normal EEG. By using PSD function, we found in event $1, \alpha$ rhythm, with wave $\alpha$ weakening in a row from back of the head, the central area, head side, front head, which is identical with normal people.

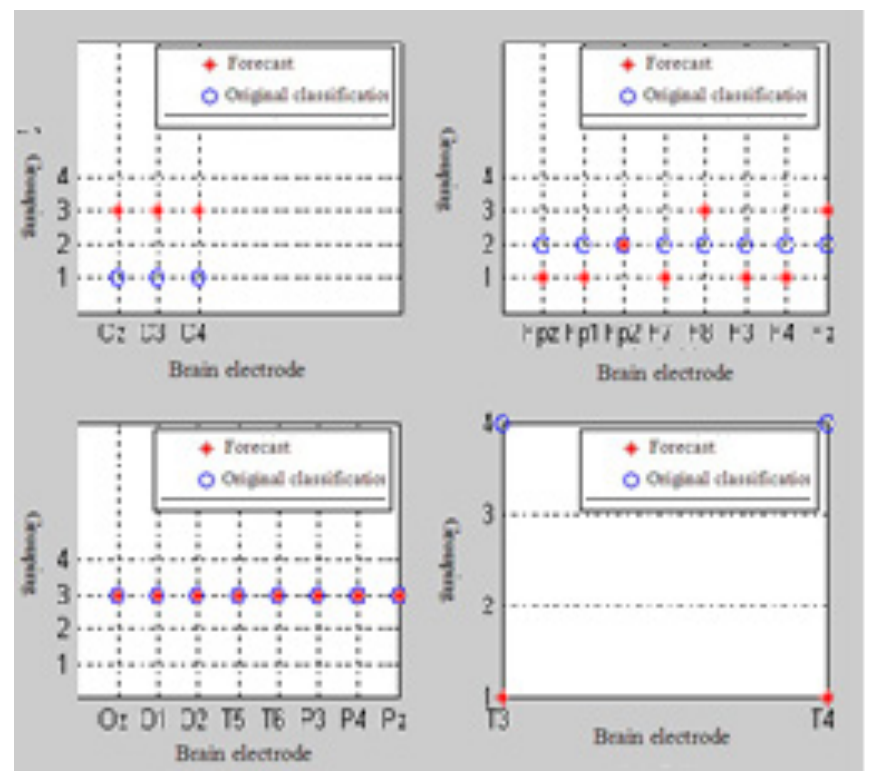

Fig. (3). Fisher results of the sixth people in Event 2.

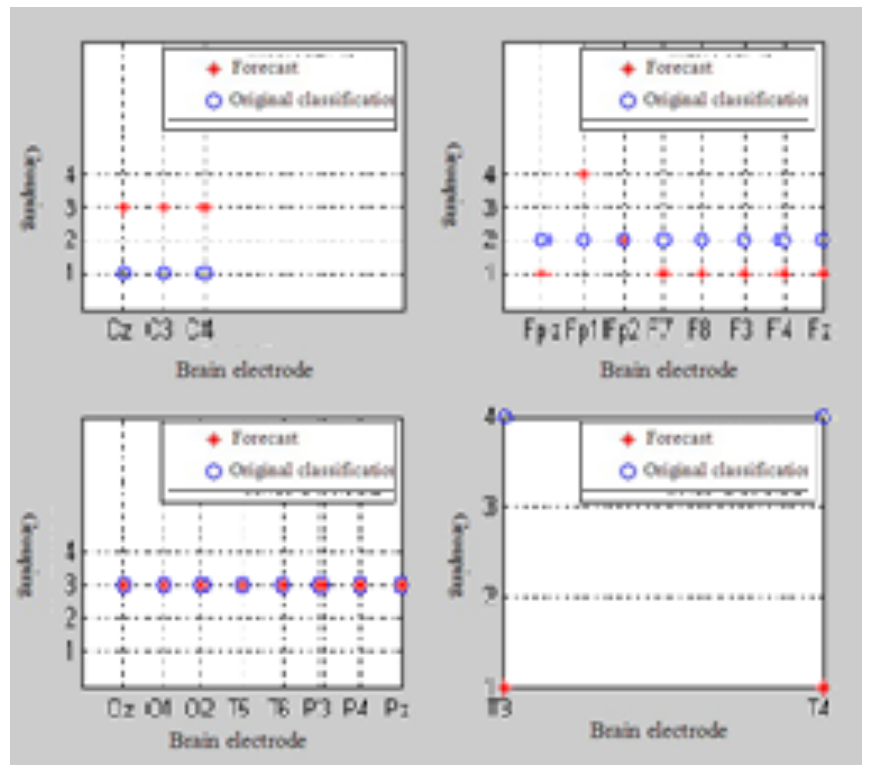

Fig. (4). Fisher results of the sixth people in Event 2.

Figs. (3 and 4) Are the Fisher's results for the sixth subject in the $2^{\text {nd }}$ event, after drinking $200 \mathrm{ml}$ of beer. In the first category, most electrodes in central area were classified at the back of the head, in the second category. Most electrodes were classified in the front of the head as the central area. In the third category, the electrode classification accuracy rate was very high. In the fourth category, most of the electrodes in the side of the head were classified as the central area.
Compared with the results of the $1^{\text {st }}$ event, the classification results changed a lot, mainly on the front, side of the head and central the area. The number of electrodes in the central area which were classified at the back increased; the number of electrodes in the front which were classified to the central increased, the number of electrodes in the side which were classified to the central increased as well. However no significant change was noticed in the electrodes classification in at back. The characteristics of EEG (wave $\alpha$ ) for normal subjects conforms to that of electrodes in the rear head, and tends to weaken gradually along the direction of the front head. After drinking $200 \mathrm{ml}$ beer, it changed a lot, and caused the change of electrode classification. The degree of brain stimulation by alcohol increased, so did the level of excitement and the degree of inhibition, while the body control reduced.

The estimated spectral density of EEG data collected after drinking $200 \mathrm{ml}$ of beer shows $\alpha$ wave in front, central and side of the head increased greatly, but not much at the back, which is identical to the results of the electrodes classification trend analysis.
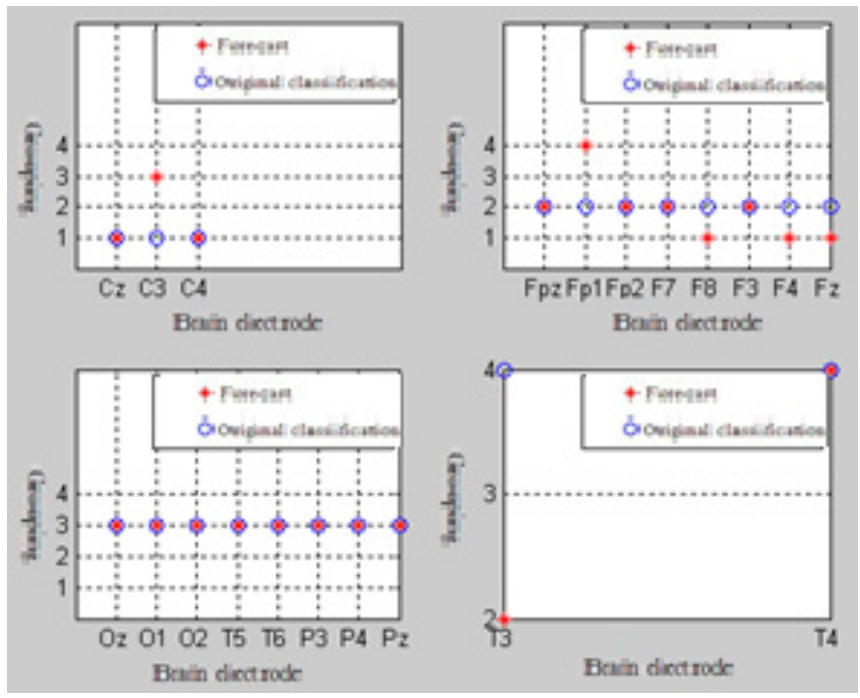

Fig. (5). Fisher results of the sixth people in Event 3.

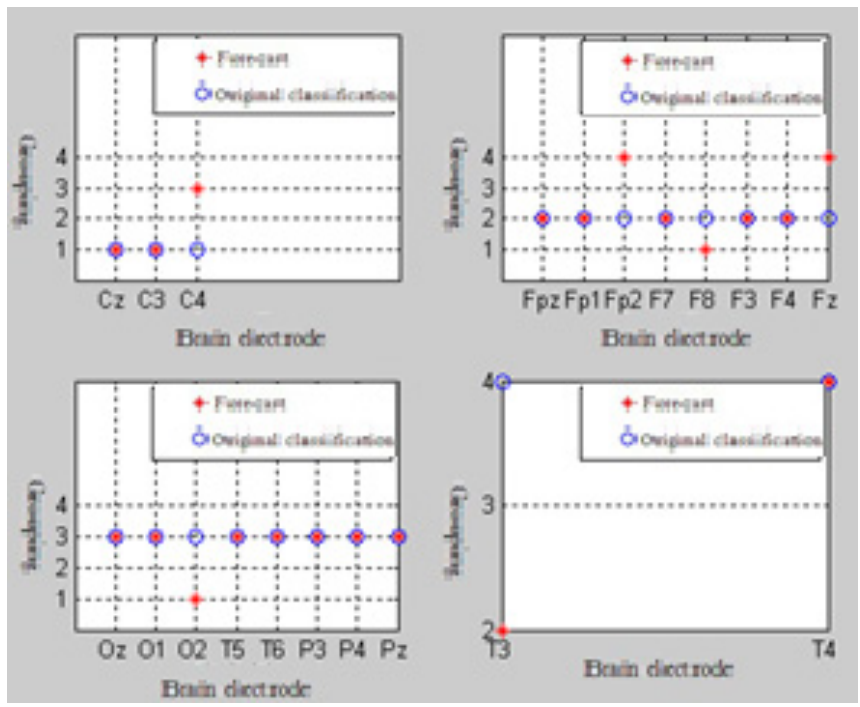

Fig. (6). Fisher results of the sixth people in Event 3 . 
Figs. (5 and 6) Are the Fisher results of the sixth people in Event 3, after drinking $400 \mathrm{ml}$ of beer. In the first category, electrodes in central area were classified as the back. In the second category, some electrodes in the front of the head were classified as the central and the side area. In the third category, the electrode classification was accurate only a few were classified as the central area. In the fourth category, some electrodes in the side of the head were classified as the front. Compared with the results of the $2^{\text {nd }}$ event, the classification results kept changing, mainly on the front side of the head and partially in central area. The number of electrodes in the central area which were classified to the back decreased; the number of electrodes in the front which were classified to the central decreased significantly; the number of electrodes in the side which were classified to the central increased; no significant change was noticed for the electrodes classification in the back. This illustrates that after drinking $400 \mathrm{ml}$ of beer, the degree of brain stimulation by alcohol decreased, so did the level of excitement and the degree of inhibition. The estimated spectral density of EEG data collected after drinking $400 \mathrm{ml}$ of beer shows a wave in front, central and side area of the head decreased slightly, but no significant change was notice in the back, which is identical to the results of the electrode classification trend analysis.

Figs. (7 and 8) Are the Fisher results of the sixth people in Event 4, after drinking $600 \mathrm{ml}$ of beer. In the first category, some electrodes in central area were classified as the front and side. In the second category, some electrodes in the front of the head were classified as central. In the third category, the electrode classification was very accurate. In the fourth category electrodes at the side of the head were classified as the front. Compared with the results of the $2^{\text {nd }}$ event, the classification results kept changing, but the changing degree reduced. The number of electrodes in the central area which were classified to the back decreased, while increased for those which were classified to the front. The number of electrodes at the front which were classified to the central area increased. The number of electrodes at the side which were classified at the front also increased. This illustrates that the degree of brain stimulation by alcohol decreased, so did the level of excitement and the degree of inhibition. The estimated spectral density of EEG data collected after drinking $600 \mathrm{ml}$ of beer shows wave in the central and side of the head decreased and the one in the front has increased slightly, which is identical to the results of the electrodes classification trend analysis.

Overall, the electrodes classification changed as the amount of alcohol taken increased, and the trend of the change was the same as that of wave $\alpha$. The change was big after drinking in the $200 \mathrm{ml}$ category, but decreased in drinking in the $600 \mathrm{ml}$ categories and electrodes classification remained stable. The change in the front, side and central area was great but only a little in the back. Fisher discriminant of EEG for the subjects 6 in different events is basically the same as that of the change of electrodes, meaning the conclusion follows a regulation and is stable.

\section{CONCLUSION}

In accordance with the strength of $\alpha$ wave, the head electrodes are divided into four species. Using 21 electrodes,
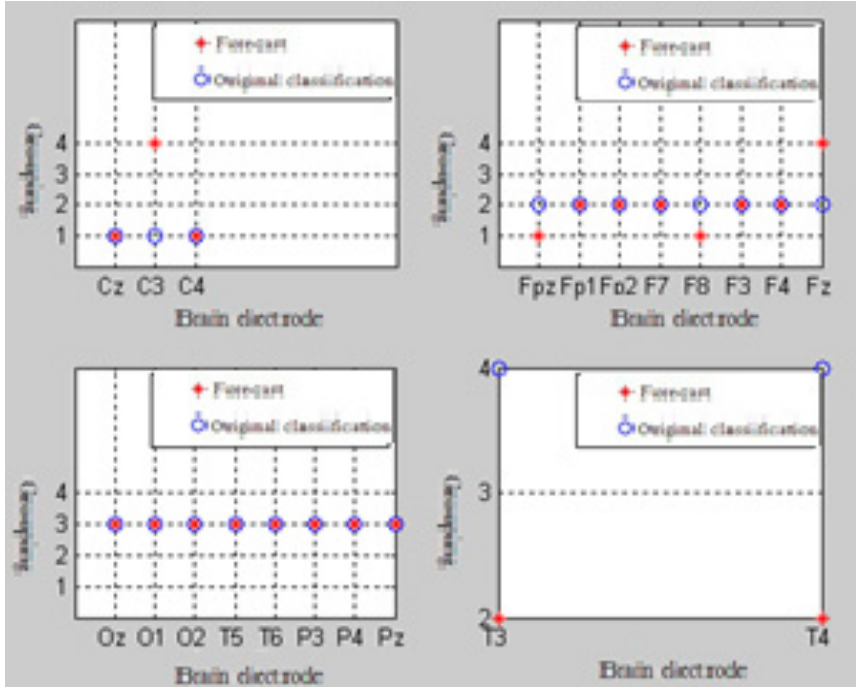

Fig. (7). Fisher results of the sixth people in Event 4.
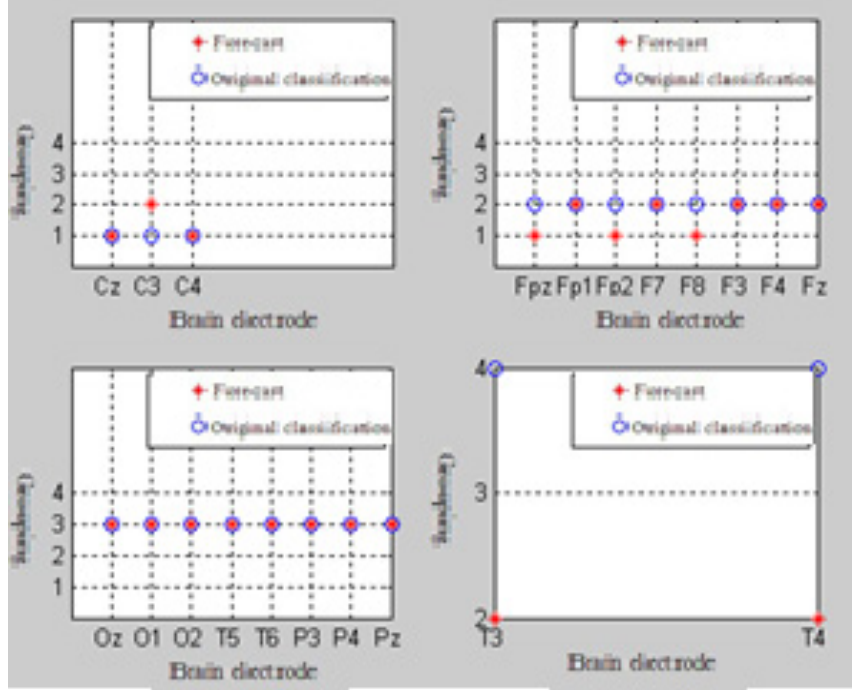

Fig. (8). Fisher results of the sixth people in Event 4.

we have performed Fisher's discriminant analysis with EEG data of six subjects in six events (no drinking event, and 3 events with $200 \mathrm{ml}$ of beer drinking after every 20 minutes). The results showed that the electrode classification changed as the alcohol intake increased. The change was big after drinking $200 \mathrm{ml}$ category, but decreased in drinking $600 \mathrm{ml}$ categories whereas the electrodes classification stayed stable. The change in the front, side and central area was found to be the greatest and it was found to be the least at the back. After drinking $200 \mathrm{ml}$ of alcohol, strong stimulation of the brain was observed, the brain became excited for a short while. As the alcohol intake was increased, the stimulation increased, the stability decreased, and the reaction of the brain was reduced.

In the 6 events, changes were not obvious in two of the men after they had beers to some extent and the EEG pointed to the no drinking status. However the electrodes classification for four of the female participants changed greatly after they had consumed $600 \mathrm{ml}$ of beers. It may be because that males were more used to the alcohol due to their drinking habits [9]. 
Details of electrodes classification in 6 events: all electrodes classification was correct in event 1 . In event 2 , the number of electrodes in the central area which were classified as the back area, increased. The number of electrodes in the front and the side which were classified as the central area also increased. In the $3^{\text {rd }}$ event, the number of electrodes in the central area which were classified to be the back area decreased. The number of electrodes in the front and the side which were classified as the central area decreased. In the $4^{\text {th }}$ event (after participants had consumed $600 \mathrm{ml}$ of beer), the number of electrodes in the central area which were classified as the back area decreased but those which were classified as the front area increased. The number of electrodes in the front which were classified as the central area decreased. The number of electrodes in the side which were classified as the front area increased. The trend of electrodes classification changes in 6 events was the same as wave $\alpha$ change trend by the estimated spectral density of EEG data collected.

\section{CURRENT \& FUTURE DEVELOPMENTS}

In this paper, we have performed Fisher's discriminant analysis on EEG data from the experiment on subjects whose drinking statuses were recorded anonymously. This data was used for feature extraction and classification decision making. In order to determine the parts of head electrode's categories after alcohol intake, in order explore the changes of EEG features. The head electrodes are divided into four species in accordance with the strength of $\alpha$ wave. In use of part of 21 electrodes EEG data of 6 subjects. We have done Fisher's discriminant analysis to EEG data of six subjects after every 20 minutes by introducing a dosage of $7.2 \mathrm{ml}$ of alcohol with six intervals forming six different events. EEG data processing and statistical analysis adopted independently designed EEG analysis toolbox and the program of correlation analysis. Results Fisher discriminant would be better applied to the future extraction and classification decisions of the EEG data.

Conclusions: EEG activity shows a significant response after alcohol intake, electrode categories are constantly changed. After drinking $200 \mathrm{ml}$ category changes are obvious, and drinking $600 \mathrm{ml}$ category changes become subtle. The changes are not so obvious in men but are relatively more obvious in women.

\section{CONFLICT OF INTEREST}

The author confirms that this article content has no conflict of interest.

\section{ACKNOWLEDGEMENTS}

Declared none.

\section{REFERENCES}

[1] H. D. Keith, "System for synchronizing EEG with auxiliary output, in particular video" U. S. Patent 20,110,184,307, July 28,2011.

[2] J. Denis, "Method for consciousness and pain monitoring, module for analyzing EEG signals, and EEG anesthesia monitor" U. S. Patent 20,140,081,094, April 26, 2014.

[3] N. Nierenberg, S. B. Wilson and M. Scheuer, "Monitoring an EEG" U. S. Patent 20,140,012,151, October 6, 1998.

[4] S. Manoli, D. Levendowski and F. Eugene, "EEG electrode and EEG electrode locator assembly" U. S. Patent 20,010,044,573, November 22, 2001

[5] D. R. Yancey, "Method and System For Displaying EEG Data" U. S. Patent 20,140,012,151, January 9,2014.

[6] S. Toyama, K. Kamitsukuri and K. Takano, "EEG measurement electrode, EEG measurement member, and EEG measurement device" Japanese Patent JP 2,013,111,361, October 6,2013.

[7] D. Hu and R. Zhang, Multivariate Data Analysis Method. Tianjin: University of Nankai Press, 1990.

[8] J. Yu and W. Yang, Multivariate statistical analysis and application. Guangzhou: University of Zhongshan Press, 2005.

[9] W. J. Williams, P. Hitten, J. Zaveri. "Time-frequency Analysis of Electrophysiology Signals in Epilesy", IEEE Transaction on Biolmedical Engineering, vol. 26, pp.133-142, 1995.

Received: September 22, 2014

Revised: November 30, 2014

Accepted: December 02, 2014

(C) Yuan Shi; Licensee Bentham Open.

This is an open access article licensed under the terms of the Creative Commons Attribution Non-Commercial License (http://creativecommons.org/licenses/by-nc/3.0/) which permits unrestricted, non-commercial use, distribution and reproduction in any medium, provided the work is properly cited. 